\title{
An unexpected neonatal outcome
}

\author{
Juan Piazze ${ }^{1 *}$, Francesco Recchia ${ }^{2}$, Massimo Bratta ${ }^{3}$, Michele Rosselli ${ }^{4}$ and Silvio Rea ${ }^{5}$ \\ ${ }^{1}$ ASL Frosinone (Casa della Salute di Ceprano-Ospedale SS Trinità di Sora), Fondazione Carlo Ferri Monterotondo, Rome, Italy \\ ${ }^{2}$ Ospedale Civile di Avezzano, Fondazione "Carlo Ferri”, Monterotondo, Rome, Italy \\ ${ }^{3}$ Ospedale Civile di Rieti, Fondazione "Carlo Ferri”, Monterotondo, Roma, Italy \\ ${ }^{4}$ Unità Operativa di Oncologia, Ospedale Civile di Frascati, Italy \\ ${ }^{5}$ Chirurgia Oncologica, Università degli Studi de L'Aquila, Dpt DISCAB, Fondazione “Carlo Ferri”, Monterotondo, Roma, Italy
}

\begin{abstract}
We report the sixth pregnancy of a 35-year-old woman, in whom mild posterior ventricular width and fetal pyelectasis were found in mid trimester ultrasound evaluation. This report describes that two mild entity soft markers may not mean an uneventful outcome, even if other unfavorable events may verify during pregnancy (i.e. IUGR in an elder pregnant woman).
\end{abstract}

\section{Introduction}

Ultrasound screening at mid pregnancy is one of the most common genetic screening used during pregnancy [1,2]. The practical concern for ultrasound screening is false-positive and false-negative (missed or not present) results. The use and understanding of ultrasound soft markers and their screening relative risks is an important option in the care of pregnant women.

An important report [3] suggested that: 1) a screening ultrasound should evaluate 8 markers, 5 of which (thickened nuchal fold, echogenic bowel, mild ventriculomegaly, echogenic focus in the heart, and choroid plexus cyst) are associated with an increased risk of fetal aneuploidy, and in some cases with nonchromosomal problems, while 3 (single umbilical artery, enlarged cisterna magna, and pyelectasis) are only associated with an increased risk of nonchromosomal abnormalities when seen in isolation, 2) identification of soft markers for fetal aneuploidy requires correlation with other risk factors, including history, maternal age, and maternal serum testing results, 3) soft markers identify a significant increase in fetal risk for genetic disease.

The case described confirms that counselling and further investigation are required to maximize management options and that two mild entity soft markers may not mean an uneventful outcome.

\section{Case report}

We report the case of a 35-year-old woman, in the course of her sixth pregnancy, with three precedent spontaneous vaginal deliveries and two spontaneous abortions, in whom posterior ventricular width and fetal pyelectasis with no uretero-calyceal dilatation were found in mid trimester ultrasound evaluation $(20+5$ wks gestation). The pregnant woman refused a suggested amniocentesis based on age and the two soft markers described. These antecedents leaded to a continuous ultrasound evaluation at $25+1,31+1$ and at $36+1$ wks gestation.

In the last ultrasound evaluation, bilateral ventriculomegaly values were found as follows: left $10.4 \mathrm{~mm}$, right $10.7 \mathrm{~mm}$ and bilateral pyelectasis: left $6.4 \mathrm{~mm}$ and right $6.1 \mathrm{~mm}$. At $36+1$ wks gestation ultrasound evaluation, an asymmetrical IUGR with no Doppler velocimetry alterations was diagnosed. Admitted, with no adverse events during hospitalization, and after eleven days (at $37+4$ wks gestation) she gave birth spontaneously a healthy $2900 \mathrm{~kg}$ male baby, with an optimal Apgar score and good neonatal conditions.

At neonatologist evaluation at birth and one week after, no uneventful neonatal outcome was diagnosed other than a slight neonatal bilateral renal pyelectasis.

\section{Conclusion}

The fetal wellbeing in one case of pregnancy with more than one soft marker found by ultrasonography, may not always mean an uneventful outcome. As described by Van den Hof MC et al (3), other than soft markers found, correlation with other risk factors, should be considered. Obviously in the case described, the pregnant's refusal to perform amniocentesis did not help in the search of an aneuploidy as more than one factor suggested (soft markers, maternal age, precedent pregnancy anamnesis).

An important report [4] suggests that despite accurate screening, there are more complex conditions in which mild ventriculomegaly (10-15 mm) can be the only non-specific finding in fetal life, making postnatal follow up mandatory.

Fetal mild bilateral pyelectasis with no calyceal dilatation is a benign condition that can be managed in the postnatal period. The initial renal pelvis diameter $(>5 \mathrm{~mm})$ and the diameter in week 31 or $35(10-15 \mathrm{~mm})$ may be valuable parameters for identifying cases that would eventually need specific postnatal procedures [5].

${ }^{*}$ Correspondence to: Dr Juan Piazze, ASL Frosinone (Casa della Salute di Ceprano-Ospedale SS Trinità di Sora), Fondazione Carlo Ferri Monterotondo, Rome, Italy, E-mail: cloudpia@me.com

Key words: ultrasound, fetal soft markers, fetal outcome

Received: June 13, 2018; Accepted: June 18, 2018; Published: June 25, 2018 
At the end, the IUGR with no maternal fetal velocimetry alterations found in one term ultrasound evaluation, had no apparent cause. Following ten days under hospital observation, and after a spontaneous delivery at $37+4$ wks gestation, she gave birth a healthy $2900 \mathrm{~kg}$ baby, who was evaluated accurately at birth and within neonatal admission, showing an uneventful whole condition.

\section{References}

1. First and Second Trimester Prenatal Screening Update [Internet]. Institute of Health Economics. Edmonton, Alberta, Canada: Institute of Health Economics (IHE); 2014 Aug.
2. First and Second Trimester Prenatal Screening for Trisomies 13, 18, and 21 and Open Neural Tube Defects [Internet]. Institute of Health Economics. Edmonton, Alberta, Canada: Institute of Health Economics (IHE); 2012 Sep.

3. Van den Hof MC, Wilson RD (2005) Diagnostic Imaging Committee, Society of Obstetricians and Gynaecologists of Canada; Genetics Committee, Society of Obstetricians and Gynaecologists of Canada. Fetal soft markers in obstetric ultrasound. J Obstet Gynaecol Can. 27:592-636.

4. Scelsa B, Rustico M, Righini A, Parazzini C, Balestriero MA, et al. (2018) Mild ventriculomegaly from fetal consultation to neurodevelopmental assessment: A single center experience and review of the literature. Eur J Paediatr Neurol. [Crossref]

5. de Paula Pereira G, Bunduki V, Hase EA, Francisco RP, Zugaib M (2016) Prenatal natural history of isolated fetal mild bilateral pyelectasis. Clinics (Sao Paulo). 71:511-516.

Copyright: ( 92018 Piazze J. This is an open-access article distributed under the terms of the Creative Commons Attribution License, which permits unrestricted use, distribution, and reproduction in any medium, provided the original author and source are credited. 